\title{
The Dark UNiverse Explorer (DUNE): proposal to ESA's cosmic vision
}

\section{A. Refregier · The DUNE Collaboration}

Received: 11 February 2008 / Accepted: 3 June 2008 / Published online: 25 July 2008 (C) Springer Science + Business Media B.V. 2008

\begin{abstract}
The Dark UNiverse Explorer (DUNE) is a wide-field space imager whose primary goal is the study of dark energy and dark matter with unprecedented precision. For this purpose, DUNE is optimised for the measurement of weak gravitational lensing but will also provide complementary measurements of baryonic accoustic oscillations, cluster counts and the Integrated Sachs Wolfe effect. Immediate auxiliary goals concern the evolution of galaxies, to be studied with unequalled statistical power, the detailed structure of the Milky Way and nearby galaxies, and the demographics of Earth-mass planets. DUNE is an Medium-class mission which makes use of readily available components, heritage from other missions, and synergy with ground based facilities to minimise cost and risks. The payload consists of a $1.2 \mathrm{~m}$ telescope with a combined visible/NIR field-of-view of $1 \mathrm{deg}^{2}$. DUNE will carry out an all-sky survey, ranging from 550 to $1600 \mathrm{~nm}$, in one visible and three NIR bands which will form a unique legacy for astronomy. DUNE will yield major advances in a broad range of fields in astrophysics including fundamental cosmology, galaxy evolution, and extrasolar planet search. DUNE was recently selected by ESA as one of the mission concepts to be studied in its Cosmic Vision programme.
\end{abstract}

The DUNE collaboration

Co-investigators: A. Refregier (PI, CEA Saclay) • M. Douspis (IAS Orsay) • Y. Mellier (IAP Paris) • B. Milliard (LAM Marseille) • P. Schneider (U. Bonn) • H.-W. Rix (MPIA) • R. Bender (MPE Garching) • F. Eisenhauer (MPE Garching) • R. Scaramella (INAF-OARM) • L. Moscardini (U. Bologna) • L. Amendola $(\mathrm{INAF-OARM}) \cdot$ F. Pasian (INAF-OATS) • F.-J. Castander (ICE, Barcelona) • M. Martinez (IFAE, Barcelona) • R. Miquel (IFAE Barcelona) •

E. Sanchez (CIEMAT Madrid) • S. Lilly (ETH Zurich) • G. Meylan (EPFL-UniGE) • M. Carollo (ETH Zurich) • F. Wildi (EPFL-UniGE) • J. Peacock (IfA Edinburgh) •

A. Refregier $(\bowtie)$

Service d'Astrophysique, CEA Saclay, bat. 709, 91191 Gif sur Yvette, France

e-mail: refregier@cea.fr 
Keywords Cosmology • Dark energy • Dark matter • Legacy survey •

Wide field imager $\cdot$ Visible and near infra-red

\section{Introduction}

Over the past decade, our large-scale view of the Universe has undergone a revolution. Cosmologists have agreed on a standard model that matches a wide range of astronomical data (eg. [14]). However, this $\Lambda \mathrm{CDM}$ concordance model relies on three ingredients whose origin and nature are unknown: dark matter, dark energy and fundamental fields driving a period of inflation, during which density fluctuations are imprinted on the Universe. All these elements of the model represent new aspects of fundamental physics, which can best be studied via astronomy. The nature of the dark energy, which now comprises the bulk of the mass-energy budget of the Universe, will determine the ultimate fate of the Universe and is among the deepest questions in physics.

S. Bridle (UCL London) • M. Cropper (MSSL) • A. Taylor (IfA Edinburgh) •

J. Rhodes (JPL) • J. Hong (JPL) • J. Booth (JPL) • S. Kahn (U. Stanford)

WG coordinators: A. Amara (CEA Saclay) · N. Aghanim (IAS Orsay) · J. Weller (UCL) •

M. Bartelmann (ZAH Heidelberg) • L. Moustakas (JPL) • R. Somerville (MPIA) •

E. Grebel (ZAH Heidelberg) • J.-P. Beaulieu (IAP Paris) • M. Della Valle (Arcetri) •

I. Hook (U. Oxford) • O. Lahav (UCL London) • A. Fontana (Roma) •

D. Bederede (CEA Saclay)

Science: F. Abdalla (UCL) • R. Angulo (Durham) • V. Antonuccio (Catane) •

C. Baccigalupi (SISSA) • D. Bacon (U. Edinburgh) • M. Banerji (UCL) • E. Bell (MPIA) •

N. Benitez (Madrid) • S. Bonometto (Milano) • F. Bournaud (CEA Saclay) •

P. Capak (Caltech) • F. Casoli (IAS Orsay) • L. Colombo (Milano) • A. Cooray (UC Irvine) •

F. Courbin (EPFL) • E. Cypriano (UCL) • H. Dahle (Oslo) • R. Ellis (Caltech) •

T. Erben (Bonn) • P. Fosalba (ICE Barcelona) • R. Gavazzi (Santa Barbara/IAP) •

E. Gaztanaga (ICE Barcelona) • A. Goobar (Stockholm U.) • A. Grazian (Obs. Roma) •

A. Heavens (U. Edinburgh) • D. Johnston (JPL) • L. King (Cambridge) • T. Kitching (Oxford) •

M. Kunz (U. Geneva) • C. Lacey (Durham) • F. Mannucci (Firenze) •

R. Maoli (Rome) • C. Magneville (CEA Saclay) • S. Matarrese (U. Padova) •

P. Melchior (ZAH Heidelberg) • A. Melchiorri (U. Roma) • M. Meneghetti (Bologna) •

J. Miralda-Escude (ICE Barcelona) • A. Omont (IAP Paris) •

N. Palanque-Delabrouille (CEA Saclay) • S. Paulin-Henriksson (CEA Saclay) •

V. Pettorino $($ SISSA) - C. Porciani (ETH Zurich) - M. Radovich $($ Obs. Napoli) - A. Rassat

$(\mathrm{UCL} / \mathrm{CEA}$ Saclay) $\cdot$ R. Saglia $(\mathrm{MPE}) \cdot$ D. Sapone (U. Geneva) •

C. Schimd (CEA Saclay) • J. Tang (UCL) • C. Tao (CPPM Marseille) • G. La Vacca (U. Milano) •

E. Vanzella (Obs. Trieste) • M. Viel (Trieste) • S. Viti (UCL) • L. Voigt (UCL) •

J. Wambsganss (ZAH Heidelberg)

Instrument: E. Atad-Ettedgui (UKATC/ROE) • E. Bertin (IAP Paris) •

O. Boulade (CEA Saclay) • I. Bryson (UKATC/ROE) • C. Cara (CEA Saclay) •

L. Cardiel (IFAE) • A. Claret (CEA Saclay) •

E. Cortina (CIEMAT) • G. Dalton (Oxford/RAL) • C. Dusmesnil (IAS Orsay) •

J.-J. Fourmond (IAS Orsay) • K. Gilmore (Stanford) • . Hofmann (MPE) •

P.-O. Lagage (CEA Saclay) • R. Lenzen (MPIA) • A. Rasmussen (Stanford) •

S. Ronayette (CEA Saclay) • S. Seshadri (JPL) • Z.H. Sun (CEA Saclay) • H. Teplitz (IPAC) •

M. Thaller (IPAC) • I. Tosh (Rutherford Lab.) • H. Vaith (MPE) • A. Zacchei (Trieste) 
The most powerful tool that can be brought to bear on these problems is weak gravitational lensing of distant galaxies; this forms the core of the DUNE mission. ${ }^{1}$ Gravitational deflection of light by intervening dark matter concentrations causes the images of background galaxies to acquire an additional ellipticity of order of a percent, which is correlated over scales of tens of arcminutes. Measuring this signature probes the expansion history in two complementary ways: (1) geometrically, through the distance-redshift relation, and (2) dynamically, through the growth rate of density fluctuations in the Universe.

Utilisation of these cosmological probes relies on the measurement of image shapes and redshifts for several billion galaxies. The measurement of galaxy shapes for weak lensing imposes tight requirements on the image quality which can only be met in the absence of atmospheric phase errors and in the thermally stable environment of space. For this number of galaxies, distances must be estimated using photometric redshifts, involving photometry measurements over a wide wavelength range in the visible and near-IR. The necessary visible galaxy colour data can be obtained from the ground, using current or upcoming instruments, complementing the unique image quality of space for the measurement of image distortions. However, at wavelengths beyond $1 \mu \mathrm{m}$, we require a wide NIR survey to depths that are only achievable from space.

Given the importance of the questions being addressed and to provide systematic cross-checks, DUNE will also measure Baryon Acoustic Oscillations, the Integrated Sachs-Wolfe effect, and galaxy Cluster Counts. Combining these independent cosmological probes, DUNE will tackle the following questions: What are the dynamics of dark energy? What are the physical characteristics of the dark matter? What are the seeds of structure formation and how did structure grow? Is Einstein's theory of general relativity the correct theory of gravity?

DUNE will combine its unique space-borne observation with existing and planned ground-based surveys, and hence increases the science return of the mission while limiting costs and risks. The panoramic visible and NIR surveys required by DUNE's primary science goals will afford unequalled sensitivity and survey area for the study of galaxy evolution and its relationship with the distribution of the dark matter, the discovery of high redshift objects, and of the physical drivers of star formation. Additional surveys at low galactic latitudes will provide a unique census of the Galactic plane and earth-mass exoplanets at distances of $0.5-5$ AU from their host star using the microlensing technique. These DUNE surveys will provide a unique all-sky map in the visible and NIR and thus complement other space missions such as Planck, WMAP, eROSITA, JWST, and WISE. The following describes the science objectives, instrument concept and mission profile (see Table 1 for a baseline summary). A description of an earlier version of the mission without NIR

\footnotetext{
${ }^{1}$ For further information on DUNE: www.dune-mission.net.
} 
Table 1 DUNE Baseline summary

\begin{tabular}{|c|c|}
\hline Science objectives & $\begin{array}{l}\text { Must: cosmology and dark energy. Should: galaxy formation } \\
\text { Could: extra-solar planets }\end{array}$ \\
\hline Surveys & $\begin{array}{l}\text { Must: } 20,000 \mathrm{deg}^{2} \text { extragalactic, Should: Full sky }\left(20,000 \mathrm{deg}^{2} \text { Galactic), }\right. \\
100 \mathrm{deg}^{2} \text { medium-deep. Could: } 4 \mathrm{deg}^{2} \text { planet hunting }\end{array}$ \\
\hline Requirements & $\begin{array}{l}1 \text { visible band }(\mathrm{R}+\mathrm{I}+\mathrm{J}) \text { for high-precision shape measurements, } \\
3 \text { NIR bands }(\mathrm{Y}, \mathrm{J}, \mathrm{H}) \text { for photometry }\end{array}$ \\
\hline Payload & $1.2 \mathrm{~m}$ telescope, Visible \& NIR cameras with $0.5 \mathrm{deg}^{2}$ FOV each \\
\hline Service module & Mars/Venus express, Gaia heritage \\
\hline Spacecraft & $2013 \mathrm{~kg}$ launch mass \\
\hline Orbit & Geosynchronous \\
\hline Launch & Soyuz S-T Fregat \\
\hline Operations & 4 year mission \\
\hline
\end{tabular}

capability and developped during a CNES phase 0 study can be found in Refregier et al. [12] and Grange et al. [13].

\section{Science objectives}

The DUNE mission will investigate a broad range of astrophysics and fundamental physics questions detailed below. Its aims are twofold: first study dark energy and measure its equation of state parameter $w$ (see definition below) and its evolution with a precision of $2 \%$ and $10 \%$ respectively, using both expansion history and structure growth, second explore the nature of dark matter by testing the cold dark matter (CDM) paradigm and by measuring precisely the sum of the neutrino masses. At the same time, it will test the validity of Einstein's theory of gravity. In addition, DUNE will investigate how galaxies form, survey all Milky-Way-like galaxies in the $2 \pi$ extra-galactic sky out to $z \sim 2$ and detect thousands of galaxies and AGN at $6<z<12$. It will provide a detailed visible/NIR map of the Milky Way and nearby galaxies and provide a statistical census of exoplanets with masses above 0.1 Earth mass and orbits greater than $0.5 \mathrm{AU}$.

\subsection{Understanding dark energy}

A variety of independent observations overwhelmingly indicate that the cosmological expansion began to accelerate when the Universe was around half of its present age. Presuming the correctness of general relativity this requires a new energy component known as dark energy. The simplest case would be Einstein's cosmological constant $(\Lambda)$, in which the dark energy density would be exactly homogeneous and independent of time. However, the description of vacuum energy from current particle physics concepts conflicts by 120 orders of magnitude with the observed value, and the discrepancy is still not understood. Cosmologists are thus strongly motivated to consider models of a dynamical dark energy, or even to contemplate modifications to general relativity. 
Explaining dark energy may well require a radical change in our understanding of Quantum Theory or Gravity, or both. One of the major aims of DUNE is to determine empirically which of these alternatives is to be preferred. The properties of dark energy can be quantified by considering its equation of state parameter $w=p / \rho c^{2}$, where $p$ and $\rho$ are its effective pressure and density. Unlike matter, dark energy has the remarkable property of having negative pressure $(w<0)$ and thus of driving the Universe into a period of accelerated expansion (if $w<-1 / 3$ ). The latter began relatively recently, around $z \leq 1$. If the dark energy resembles a cosmological constant $(w=-1)$, it can only be directly probed in the low-redshift Universe (see Fig. 1). This expansion history can be measured in two distinct ways (see Fig. 1): (1) the distanceredshift relation $D(z)$; (2) the growth of structure (i.e. galaxies and clusters of galaxies). The $D(z)$ relation can be probed geometrically using 'standard candles' such as supernovae, or via measures of the angular diameter distance from gravitational lensing or from the "standard rod" of Baryon Acoustic Oscillations (BAO). The accelerated expansion slows down the gravitational growth of density fluctuations; this growth of structure can be probed by comparing the amplitude of structure today relative to that when the CMB was formed. Many models for dark energy and modifications to gravity have been proposed in which the equation of state parameter $w$ vary with time. A convenient approximation is a linear dependence on the scale factor $a=$ 1/(1+z): $w(a)=w_{n}+\left(a_{n}-a\right) w_{a}$, where $w_{n}$ is the value of the equation of state at a pivot scale factor $a_{n}$ (close to 0.6 for most probes) and $w_{a}$ describes the redshift evolution. The goal of future surveys is to measure $w_{n}$ and $w_{a}$ to high precision. To judge their relative strengths we use a standard dark energy figure of merit (FoM) [2], which we define throughout this proposal as: FoM $=1 /\left(\Delta w_{n} \Delta w_{a}\right)$, where $\Delta w_{n}$ and $\Delta w_{a}$ are the $(1 \sigma)$ errors on the equation of state parameters. This FoM is inversely proportional to the area of the error ellipse in the $\left(w_{n}-w_{a}\right)$ plane.

\subsection{DUNE's cosmological probes}

DUNE will deduce the expansion history from the two methods, distanceredshift relation and growth of structure. DUNE has thus the advantage of probing the parameters of dark energy in two independent ways. A single accurate technique can rule out many of the suggested members of the family of dark energy models, but it cannot test the fundamental assumptions about gravity theory. If general relativity is correct, then either $D(z)$ or the growth of structure can determine the expansion history. In more radical models that violate general relativity, however, this equivalence between $D(z)$ and growth of structure does not apply (see Fig. 1). For this purpose, DUNE will use a combination of the following cosmological probes. The precision on Dark Energy parameters achieved by DUNE's weak lensing survey and complementary probes described below is shown in Fig. 2 and Table 2. 

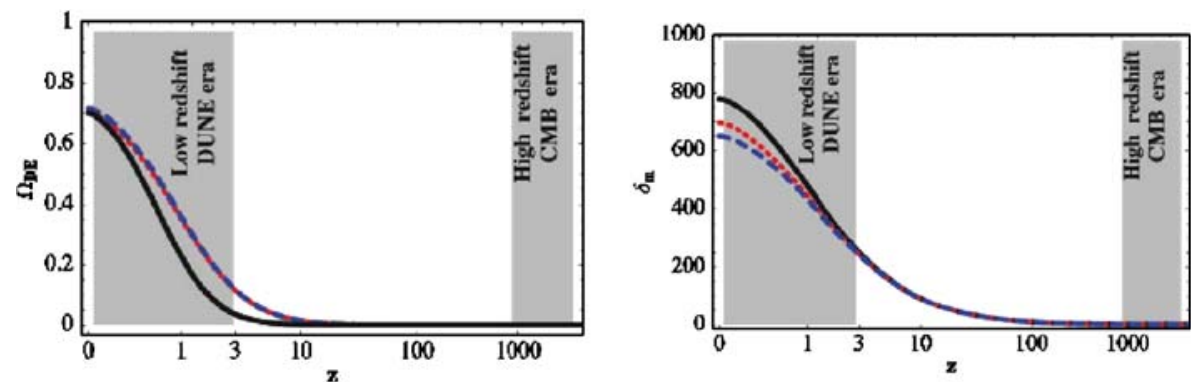

Fig. 1 Effects of dark energy. Left fraction of the density of the universe in the form of dark energy as a function of redshift $z$, for a model with a cosmological constant $(w=-1$, black solid line), dark energy with a different equation of state $(w=-0.7$, red dotted line $)$, and a modified gravity model (blue dashed line). Dark energy becomes dominant in the low redshift universe era probed by DUNE, while the early Universe is probed by the CMB. Right growth factor of structures for the same models. Only by measuring the geometry (left panel) and the growth of structure (right panel) at low redshifts can a modification of dark energy be distinguished from that of gravity. Weak lensing measures both effects

Weak Lensing-A Dark Universe Probe: As light from galaxies travels towards us, its path is deflected by the intervening mass density distribution, causing the shapes of these galaxies to appear distorted by a few percent. The weak lensing method measures this distortion by correlating the shapes of background galaxies to probe the density field of the Universe. By dividing galaxies into redshift (or distance) bins, we can examine the growth of structure and make three-dimensional maps of the dark matter. An accurate lensing survey, therefore, requires precise measurements of the shapes of galaxies
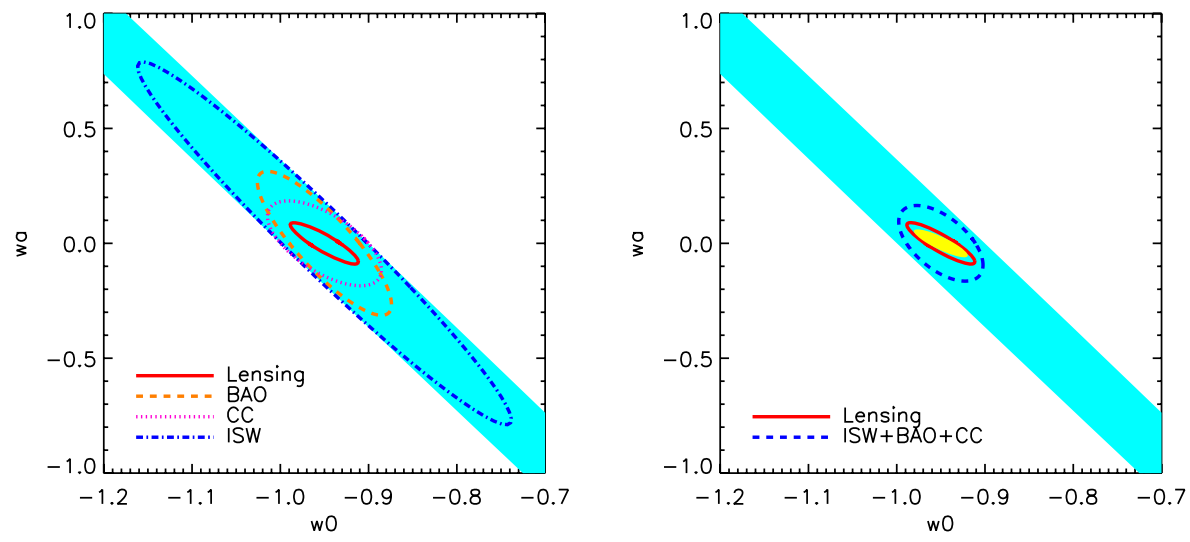

Fig. 2 Left expected errors on the dark energy equation of state parameters for the four probes used by DUNE (68\% statistical errors). The light blue band indicates the expected errors from Planck. Of the four methods, weak lensing clearly has the greatest potential. Right the combination of BAO, CC and ISW (red solid line) begins to reach the potential of the lensing survey (blue dashed line) and provides an additional cross-check on systematics. The yellow ellipse corresponds to the combination of all probes and reaches a precision on dark energy of $2 \%$ on $w_{n}$ and $10 \%$ on $w_{a}$ 
Table 2 Dark energy and initial conditions figures of merit for DUNE and Planck

\begin{tabular}{|c|c|c|c|c|c|c|}
\hline & \multicolumn{2}{|c|}{ Dark energy sector } & \multicolumn{2}{|c|}{ Initial conditions sector } & \multirow{2}{*}{$\frac{\mathrm{DE}}{\mathrm{FoM}}$} & \multirow{2}{*}{$\begin{array}{l}\text { IC } \\
\text { FoM }\end{array}$} \\
\hline & $\overline{\Delta w_{n}}$ & $\Delta w_{a}$ & $\overline{\Delta \sigma_{8}}$ & $\Delta n_{s}$ & & \\
\hline Planck & 0.03 & 3 & 0.03 & 0.004 & 11 & 8,000 \\
\hline DUNE & 0.02 & 0.1 & 0.006 & 0.01 & 500 & 17,000 \\
\hline DUNE + Planck & 0.01 & 0.05 & 0.002 & 0.003 & 2000 & 170,000 \\
\hline \multicolumn{5}{|c|}{ Factor improvement of DUNE + Planck over Planck only } & 180 & 20 \\
\hline
\end{tabular}

as well as information about their redshifts. High-resolution images of large portions of the sky are required, with low levels of systematic errors that can only be achieved via observations from a thermally stable satellite in space. Analyses of the dark energy require precise measurements of both the cosmic expansion history and the growth of structure. Weak lensing stands apart from all other available methods because it is able to make accurate measurements of both effects. Given this, the optimal dark energy mission (and dark sector mission) is one that is centred on weak gravitational lensing and is complemented by other dark energy probes.

Baryon Acoustic Oscillations (BAO)—An Expansion History Probe: The scale of the acoustic oscillations caused by the coupling between radiation and baryons in the early Universe can be used as a 'standard ruler' to determine the distance-redshift relation. Using DUNE, we can perform BAO measurements using photometric redshifts yielding the three-dimensional positions of a large sample of galaxies. All-sky coverage in the NIR enabled by DUNE, impossible from the ground, is crucial to reach the necessary photometric redshift accuracy for this BAO survey.

Cluster Counts (CC)-A Growth of Structure Probe: Counts of the abundance of galaxy clusters (the most massive bound objects in the Universe) as a function of redshift are a powerful probe of the growth of structure. There are three ways to exploit the DUNE large-area survey, optimised for weak lensing, for cluster detection: strong lensing; weak lensing; and optical richness.

Integrated Sachs-Wolfe (ISW) Effect-A Higher Redshift Probe: The ISW effect is the change in CMB photon energy as it passes through a changing potential well. Its presence indicates either space curvature, a dark energy component or a modification to gravity. The ISW effect is measured by cross-correlating the $\mathrm{CMB}$ with a foreground density field covering the entire extra-galactic sky, as measured by DUNE. Because it is a local probe of structure growth, ISW will place complementary constraints on dark energy, at higher redshifts, relative to the other probes [6]. 


\subsection{Understanding dark matter}

Besides dark energy, one major component of the concordance model of cosmology is dark matter ( $\sim 90 \%$ of the matter in the Universe, and $\sim 25 \%$ of the total energy). The standard assumption is that the dark matter particle(s) is cold and non-collisional (CDM). Besides direct and indirect dark matter detection experiments, its nature may well be revealed by experiments such as the large hadron collider (LHC) at CERN, but its physical properties may prove to be harder to pin down without astronomical input. one way of testing this is to study the amount of substructure in dark matter halos on scales 1$100^{\prime \prime}$, which can be done using high order galaxy shape measurements and strong lensing with DUNE. Weak lensing measurements can constrain the total neutrino mass and number of neutrino species through observations of damping of the matter power spectrum on small scales. Combining DUNE measurements with Planck data would reduce the uncertainty on the sum of neutrino masses to $0.04 \mathrm{eV}$, and may therefore make the first measurement of the neutrino mass [8].

\subsection{Understanding the seeds of structure formation}

It is widely believed that cosmic structures originated from vacuum fluctuations in primordial quantum fields stretched to cosmic scales in a brief period during inflation. In the most basic inflationary models, the power spectrum of these fluctuations is predicted to be close to scale-invariant, with a spectral index $n_{s}$ and amplitude parameterised by $\sigma_{8}$. As the Universe evolved, these initial fluctuations grew. $\mathrm{CMB}$ measurements probe their imprint on the radiation density at $z \sim 1,100$. Density fluctuations continued to grow into the structures we see today. Weak lensing observations with DUNE will lead to a factor of 20 improvement on the initial conditions as compared to CMB alone (see Table 2).

\subsection{Understanding Einstein's gravity}

Einstein's General Theory of Relativity, the currently accepted theory of gravity, has been well tested on solar system and galactic scales. Various modifications to gravity on large scales (e.g. by extra dimensions, superstrings, non-minimal couplings or additional fields) have been suggested to avoid dark matter and dark energy. The weak lensing measurements of DUNE will be used to test the underlying theory of gravity, using the fact that modified gravity theories typically alter the relation between geometrical measures and the growth of structure (see Fig. 1). DUNE can measure the growth factor exponent $\gamma$ with a precision of $2 \%$.

Meeting the above cosmological objectives necessitates an extra-galactic allsky survey (DASS-EX) in the visible/NIR with galaxies at a median redhift of $z \sim 1$. To this survey, will be added a shallower survey of the Galactic plane (DASS-G) which will complete the coverage to the full sky, as well 
as a medium-deep survey of $100 \mathrm{deg}^{2}$ (DUNE-MD) and a pencil beam microlensing survey for planets in the Galactic bulge.

Focussed on the dark sector, DUNE will produce an invaluable broad survey legacy. DASS will cover a 10,000 times larger area than other optical/nearIR surveys of the same or better resolution, will be $4 \mathrm{mag}$ deeper than the GAIA photometry and six times higher resolution than the SDSS. In the infrared, DASS-EX will be nearly 1,000 times deeper (in J) than the all-sky 2MASS survey with an effective search volume which will be 5,000-fold that of the UKIDDS large area survey currently underway, and 500-fold that of the proposed VISTA Hemisphere Survey. It would take VISTA 8,000 years to match DASS-EX depth and 20,000 deg ${ }^{2}$ area coverage. DASS-MD will bridge the gap between DASS-EX and expected JWST surveys.

\subsection{Tracking the formation of galaxies and AGN with DUNE}

While much progress has been made in understanding the formation of large scale structure, there are still many problems in forming galaxies within this structure with the observed luminosity function and morphological properties. This is now a major problem in astronomy. Obtaining deep high spatial resolution near-IR images will be central to the study galaxy morphology and clustering. A large area survey is required for rare but important events, such as the merger rate of very massive galaxies. DUNE will deliver this key capability.

Using DUNE's weak lensing maps, we will study the relationship between galaxy mass and light, the bias, by mapping the total mass density and the stellar mass and luminosity. Galaxy clusters are the largest scale signposts of structure formation. While at present only a few massive clusters at $z>1$ are known, DUNE will find hundreds of Virgo-cluster-mass objects at $z>2$, and several thousand clusters of $\mathrm{M}=1-2 \times 10^{13} \mathrm{M}_{\text {sun }}$. The latter are the likely environments in which the peak of QSO activity at $z \sim 2$ takes place, and hold the empirical key to understanding the heyday of QSO activity.

Using the Lyman-dropout technique in the near-IR, the DUNE-MD survey will be able to detect the most luminous objects in the early Universe $(z>6): \sim 10^{4}$ star-forming galaxies at $z \sim 8$ and up to $10^{3}$ at $z \sim 10$, for SFRs $>30-100 \mathrm{M}_{\odot} / \mathrm{yr}$. It will also be able to detect significant numbers of high- $z$ quasars: up to $10^{4}$ at $z \sim 7$, and $10^{3}$ at $z \sim 9$. These will be central to understanding the reionisation history of the Universe.

Dune will also detect a very large number of strong lensing systems: about $10^{5}$ galaxy-galaxy lenses, $10^{3}$ galaxy-quasar lenses and 5,000 strong lensing arcs in clusters (see [9]). It is also estimated that several tens of galaxy-galaxy lenses will be double Einstein rings [7], which are powerful probes of the cosmological model as they simultaneously probe several redshifts.

In addition, during the course of the DUNE-MD survey (over 6 months), we expect to detect $\sim 3,000$ Type Ia Supernovae with redshifts up to $z \sim 0.6$ and a comparable number of Core Collapse SNe (Types II and Ib/c) out to $z \sim 0.3$. 
This will lead to measurement of SN rates thus providing information on their progenitors and on the star formation history.

\subsection{Studying the Milky Way with DUNE}

DUNE is also primed for a breakthrough in Galactic astronomy. DASS-EX, complemented by the shallower survey of the Galactic plane (with $|b|<30$ deg) will provide all-sky high resolution $\left(0.23^{\prime \prime}\right.$ in the wide red band, and $0.4^{\prime \prime}$ in YJH) deep imaging of the stellar content of the Galaxy, allowing the deepest detailed structural studies of the thin and thick disk components, the bulge/bar, and the Galactic halo (including halo stars in nearby galaxies such as M31 and M33) in bands which are relatively insensitive to dust in the Milky Way.

DUNE will be little affected by extinction and will supersede by orders of magnitude all of the ongoing surveys in terms of angular resolution and sensitivity. DUNE will thus enable the most comprehensive stellar census of late-type dwarfs and giants, brown dwarfs, He-rich white dwarfs, along with detailed structural studies, tidal streams and merger fragments. DUNE's sensitivity will also open up a new discovery space for rare stellar and lowtemperature objects via its H-band imaging. Currently, much of Galactic structure studies are focussed on the halo. Studying the Galactic disk components requires the combination of spatial resolution (crowding) and dust-penetration (H-band) that DUNE can deliver.

Beyond our Milky Way, DUNE will also yield the most detailed and sensitive survey of structure and substructure in nearby galaxies, especially of their outer boundaries, thus constraining their merger and accretion histories.

\subsection{Search for exo-planets}

The discovery of extrasolar planets is the most exciting development in astrophysics over the past decade, rivalled only by the discovery of the acceleration of the Universe. Space observations (e.g. COROT, KEPLER), supported by ground-based high-precision radial velocity surveys will probe low-mass planets (down to $1 M_{\oplus}$ ). DUNE is also perfectly suited to trace the distribution of matter on very small scales those of the normally invisible extrasolar planets. Using microlensing effect, DUNE can provide a statistical census of exoplanets in the Galaxy with masses over $0.1 M_{\oplus}$ from orbits of 0.5 AU to free-floating objects. This includes analogues to all the solar system's planets except for Mercury, as well as most planets predicted by planet formation theory. Microlensing is the temporary magnification of a Galactic bulge source star by the gravitational potential of an intervening lens star passing near the line of sight. A planet orbiting the lens star, will have an altered magnification, showing a brief flash or a dip in the observed light curve of the star (see Fig. 3). Because of atmospheric seeing (limiting the monitoring to large source stars), and poor duty cycle even using networks, ground-based microlensing surveys are only able to detect a few to $15 M_{\oplus}$ planets in the vicinity of the Einstein ring radius (2-3 AU). The high angular resolution of DUNE, and 
Fig. 3 Exoplanet discovery parameter space (planet mass vs orbit size) showing for reference the eight planets from our solar system (labeled as letters), those detected by Doppler wobble $(\mathrm{T})$, transit (circle), and microlensing. We outline regions that can be probed by different methods. Note the uniqueness of the parameter space probed by DUNE compared to other techniques

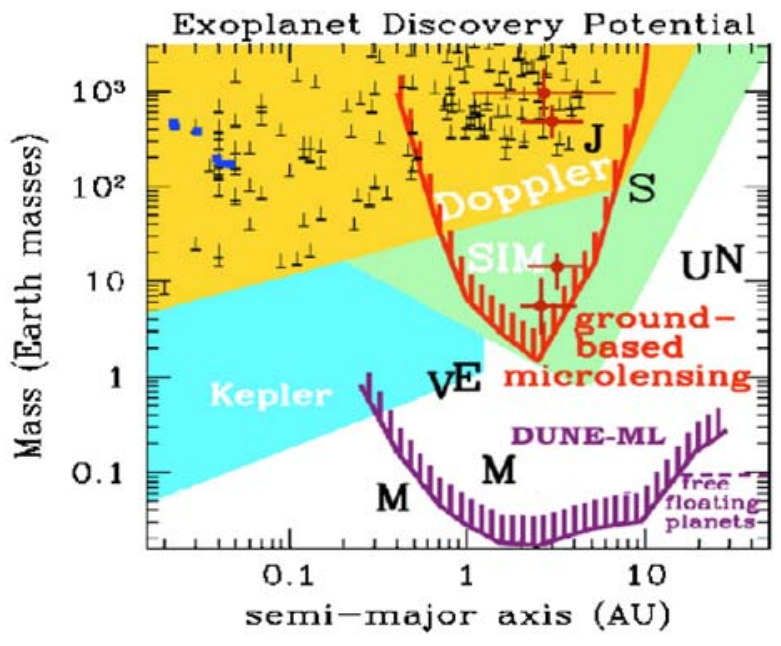

the uninterrupted visibility and NIR sensitivity afforded by space observations will provide detections of microlensing events using as sources $\mathrm{G}$ and $\mathrm{K}$ bulge dwarfs stars and therefore can detect planets down to $0.1-1 M_{\odot}$ from orbits of 0.5 AU. Moreover, there will be a very large number of transiting hot Jupiters detected towards the Galactic bulge as 'free' ancillary science. A space-based microlensing survey is thus the only way to gain a comprehensive census and understanding of the nature of planetary systems and their host stars. We also underline that the planet search scales linearly with the surface of the focal plane and the duration of the experiment.

\section{DUNE surveys: the need for all-sky imaging from space}

There are two key elements to a high precision weak lensing survey: a large survey area to provide large statistics, and the control of systematic errors. Figure 4 shows that to reach our dark energy target ( $2 \%$ error on $w_{n}$ ) a survey of 20,000 square degrees with galaxies at $z \sim 1$ is required. This result is based on detailed studies showing that, for a fixed observing time, the accuracy of all the cosmological parameters is highest for a wide rather than deep survey $[3,4]$. This required survey area drives the choice of a $1.2 \mathrm{~m}$ telescope and a combined visible/NIR FOV of $1 \mathrm{deg}^{2}$ for the DUNE baseline.

Ground based facilities plan to increase area coverage, but they will eventually be limited by systematics inherent in ground based observations (atmospheric seeing which smears the image, instabilities of ground based PSFs, telescope flexure and wind-shake, and inhomogeneous photometric calibrations arising from seeing fluctuations). The most recent ground-based wide field imagers (e.g. MegaCam on CFHT, and Subaru) have a stochastic variation of the PSF ellipticity of the order of a few percent, i.e. of the 

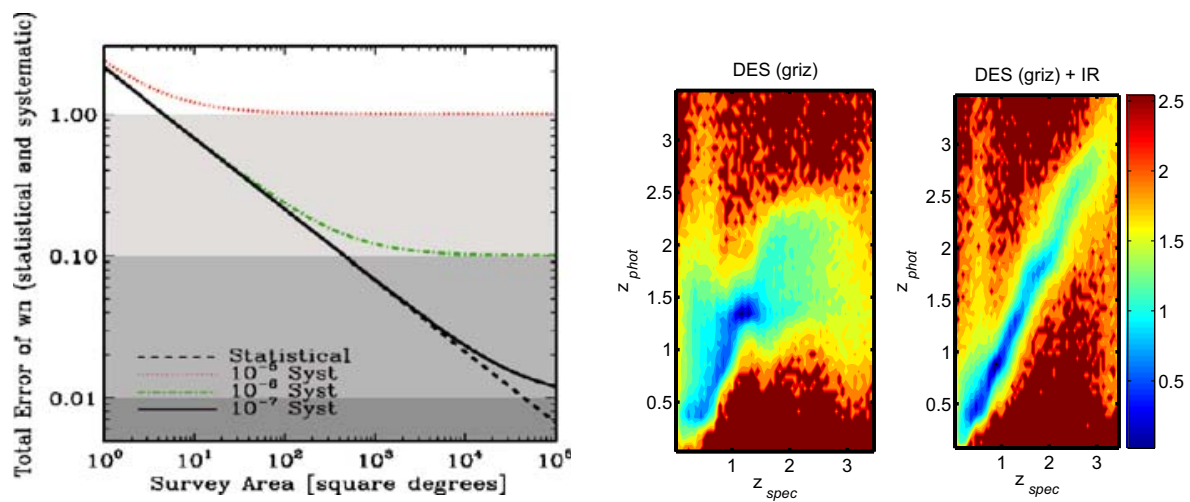

Fig. 4 Left error on the dark energy equation of state parameter $w_{n}$ as a function of weak lensing survey area (in $\mathrm{deg}^{2}$ ) for several shape measurement systematic levels (assuming 40 galaxies/amin ${ }^{2}$ with a median redshift $z_{m}=1$ ). An area of $20,000 \mathrm{deg}^{2}$ and a residual systematic shear variance of $\sigma_{\text {sys }}^{2}<10^{-7}$ is required to achieve the DUNE objective (error on $w_{n}$ better than $2 \%$ ). Right (from [1]): Photometric redshift performance for a DES-like ground survey with and without the DUNE NIR bands $(\mathrm{J}, \mathrm{H})$. The deep NIR photometry, only achievable in space, results in a dramatic reduction of the photometric redshift errors and catastrophic failures which are needed for all the probes (weak lensing, BAO, CC, ISW)

same order of magnitude as the sought-after weak lensing signal. Current measurements have a residual shear systematics variance of $\sigma_{\text {sys }}^{2} \sim 10^{-5}$, as indicated with both the results of the STEPII program and the scatter in measured value of $\sigma_{8}$. This level of systematics is comparable to the statistical errors for surveys that cover a few tens of square degree (Fig. 4). As seen on the figure, to reach DUNE's dark energy targets, the systematics must be at the level of $\sigma_{\text {sys }}^{2} \sim 10^{-7}, 100$ times better than the current level (see [4] for details). While ground based surveys may improve their systematics control, reaching this level will be an extreme challenge. One ultimate limit arises from the finite information contained in the stars used to calibrate the PSF, due to noise and pixelisation. Simulations by Paulin-Henriksson et al. [10] show that, to reach our systematic targets, the PSF must remain constant (within a tolerance of $0.1 \%$ ) over $50 \operatorname{arcmin}^{2}$ (which corresponds to $\sim 50$ stars). While this is prohibitive from the ground, we have demonstrated during a CNES phase 0 study [12], that with the careful design of the instrument, this can be achieved from space. In addition to shape measurements, wide area imagining surveys use photometric information to measure the redshift of galaxies in the images. Accurate measurements of the photometric redshifts require the addition of NIR photometry (an example of this is shown in Fig. 4, right panel, and also Abdalla et al. [1]). Such depths in the NIR cannot be achieved from the ground over wide area surveys and can only be done from space. 
Table 3 Requirements and geometry for the four DUNE surveys

\begin{tabular}{|c|c|c|c|}
\hline \multicolumn{4}{|c|}{ Wide Extragalactic Survey DASS-EX (must) } \\
\hline \multicolumn{2}{|c|}{ Area } & \multirow{2}{*}{\multicolumn{2}{|c|}{$\begin{array}{c}20,000 \text { sq degrees }-|b|>30 \mathrm{deg} \\
>20 \mathrm{deg} \times 20 \mathrm{deg}\end{array}$}} \\
\hline \multirow{2}{*}{ Survey Strategy } & Contiguous patches & & \\
\hline & Overlap & \multicolumn{2}{|r|}{$10 \%$} \\
\hline \multicolumn{2}{|c|}{ Shape Measurement Channel } & $\mathrm{R}+\mathrm{I}+\mathrm{Z}(550-920 \mathrm{~nm})$ & $\mathrm{R}+\mathrm{I}+Z_{A B}<24.5(10 \sigma \mathrm{ext})$ \\
\hline \multirow{3}{*}{\multicolumn{2}{|c|}{ Photometric Channel }} & $\mathrm{Y}(920-1146 \mathrm{~nm})$ & $Y_{A B}<24(5 \sigma$ point $)$ \\
\hline & & $\mathrm{J}(1146-1372 \mathrm{~nm})$ & $J_{A B}<24(5 \sigma$ point $)$ \\
\hline & & $\mathrm{H}(1372-1600 \mathrm{~nm})$ & $H_{A B}<24(5 \sigma$ point $)$ \\
\hline \multirow{2}{*}{ PSF } & Size \& Sample & $0.23 "$ FWHM & $>2.2$ pixels per $\mathrm{FWHM}$ \\
\hline & Stability & \multicolumn{2}{|c|}{ within tolerance of 50 stars } \\
\hline \multirow{2}{*}{ Image Quality } & Dead pixels & \multicolumn{2}{|c|}{$<5 \%$ of final image } \\
\hline & Linearity & \multicolumn{2}{|c|}{ Instrument calibratable for $1<\mathrm{S} / \mathrm{N}<1000$} \\
\hline \multicolumn{4}{|c|}{ Medium Deep Survey DUNE-MD (should) } \\
\hline \multicolumn{2}{|c|}{ Area } & \multicolumn{2}{|c|}{$\sim 100$ sq degrees - Ecliptic poles } \\
\hline Survey Strategy & Contiguous patches & \multicolumn{2}{|c|}{ Two patches each $7 \mathrm{deg} \times 7 \mathrm{deg}$} \\
\hline \multicolumn{2}{|c|}{ Photometric Channel } & \multicolumn{2}{|c|}{$Y_{A B}, J_{A B}, H_{A B}<26(5 \sigma$ point $)$ - for stack } \\
\hline \multicolumn{2}{|c|}{ PSF } & \multicolumn{2}{|c|}{ Same conditions as the wide survey } \\
\hline \multicolumn{4}{|c|}{ Wide Galactic Survey DASS-G (should) } \\
\hline \multicolumn{2}{|c|}{ Area } & \multicolumn{2}{|c|}{20,000 sq degrees $-|b|<30 \mathrm{deg}$} \\
\hline \multicolumn{2}{|c|}{ Shape Measurement Channel } & \multicolumn{2}{|c|}{$R+I+Z_{A B}<23.8(5 \sigma$ ext $)$} \\
\hline \multicolumn{2}{|c|}{ Photometric Channel } & \multicolumn{2}{|c|}{$Y_{A B}, J_{A B}, H_{A B}<22(5 \sigma$ point $)$} \\
\hline PSF & Size & & 3" FWHM \\
\hline \multicolumn{4}{|c|}{ Microlensing Survey DUNE-ML (could) } \\
\hline \multicolumn{2}{|c|}{ Area } & \multicolumn{2}{|c|}{4 sq degrees - Galactic bulge } \\
\hline Survey Strategy & Time sampling & \multirow{3}{*}{\multicolumn{2}{|c|}{$\begin{array}{c}\text { Every } 20 \text { min }-1 \text { month blocks }- \text { total of } 3 \text { months } \\
Y_{A B}, J_{A B}, H_{A B}<22(5 \sigma \text { point })-\text { per visit } \\
<0.4 " \text { FWHM }\end{array}$}} \\
\hline \multicolumn{2}{|c|}{ Photometric Channel } & & \\
\hline PSF & Size & & \\
\hline
\end{tabular}

To achieve the scientific goals listed in Section 2, DUNE will perform four surveys detailed in the following and in Table 3.

\subsection{Wide extragalactic survey: DASS-EX}

To measure dark energy to the required precision, we need to make measurements over the entire extra-galactic sky to a depth which yields $40 \mathrm{gal} / \mathrm{arcmin}^{2}$ useful for lensing with a median redshift $z_{m} \simeq 0.9$. This can be achieved with a survey (DASS-EX) that has AB-magnitude limit of 24.5 (10 $\sigma$ extended source) in a broad red visible filter $(\mathrm{R}+\mathrm{I}+\mathrm{Z})$. Based on the fact that DUNE focuses on observations that cannot be obtained from the ground, the wide survey relies on two unique factors that are enabled by space: image quality in the visible and NIR photometry. Central to shape measurements for weak lensing the PSF of DUNE needs to be sampled better than 2-2.5 pixels per FWHM [10], to be constant over 50 stars around each galaxy (within a tolerance of $\sim 0.1 \%$ in shape parameters), and to have a wavelength dependence which can be calibrated. Accurate measurement of the redshift of distant galaxies $(z \sim 1)$ requires photometry in the NIR where galaxies have a distinctive feature (the 4,000 A break). Deep NIR photometry requires space observations. The bands 
$\mathrm{Y}, \mathrm{J}$ and $\mathrm{H}$ are the perfect synergy for ground based survey complement (see [1] for discussion), as recommended by the ESO/ESA Working Group on Fundamental Cosmology [11].

\subsection{Legacy surveys: DASS-G, DUNE-MD, and DUNE-ML}

We propose to allocate six months to a medium deep survey (DUNE-MD) with an area of $100 \mathrm{deg}^{2}$ to magnitudes of 26 in $\mathrm{Y}, \mathrm{J}$ and $\mathrm{H}$, located at the $\mathrm{N}$ and $\mathrm{S}$ ecliptic poles. This survey can be used to calibrate DUNE during the mission, by constructing it from a stack of $>30$ sub-images too achieve the required depths. DUNE will also perform a wide Galactic survey (DASS-G) that will complement the $4 \pi$ coverage of the sky and a microlensing survey (DUNE-ML). Both surveys require short exposures. Together with the DASSEX, these surveys need good image quality with low level of stray light. A summary of all the surveys is shown in Table 3.

\section{Mission profile and payload instrument}

The mission design of DUNE is driven by the need for the stability of the PSF and large sky coverage. PSF stability puts stringent requirements on pointing and thermal stability during the observation time. The 20,000 square degrees of DASS-EX demands high operational efficiency, which can be achieved using a drift scanning mode (or Time Delay Integration, TDI, mode) for the CCDs in the visible focal plane. TDI mode necessitates the use of a counter-scanning mirror to stabilize the image in the NIR focal plane channel.

The baseline for DUNE is a Geosynchronous Earth orbit (GEO), with a low inclination and altitude close to a standard geostationary orbit. Based on Phase 0 CNES study, this solution was chosen to meet both the high science telemetry needs and the spacecraft low perturbation requirements. This orbit also provides substantial launch flexibility, and simplifies the ground segment.

As for the PSF size and sampling requirements, a baseline figure for the line-of-sight stability is 0.5 pixel (smearing MTF $>0.99$ at cut-off frequency), with the stability budget to be shared between the telescope thermal stability (0.35 pixel) and the attitude orbit control system (AOCS) (0.35 pixel). This implies a line-of-sight stability better than $0.2 \mu \mathrm{rad}$ over $375 \mathrm{~s}$ (the integration time across one CCD). This stringent requirement calls for a minimalization of external perturbations which mainly consist of solar radiation pressure and gravity gradient torques. A gravitational torque of $20 \mu \mathrm{Nm}$ is acceptable, and requires an orbit altitude of at least $25,000 \mathrm{~km}$. The attitude and orbit control design is based on proportional actuators.

A stable thermal environment is requested for the payload $(\sim 10 \mathrm{mK}$ variation over $375 \mathrm{~s}$ ), hence mission design requires a permanent cold face for the focal plane radiators and an orbit that minimizes heat load from the Earth. This could be achieved by having the whole payload in a regulated temperature cavity. 
Fig. 5 Overview of all payload elements

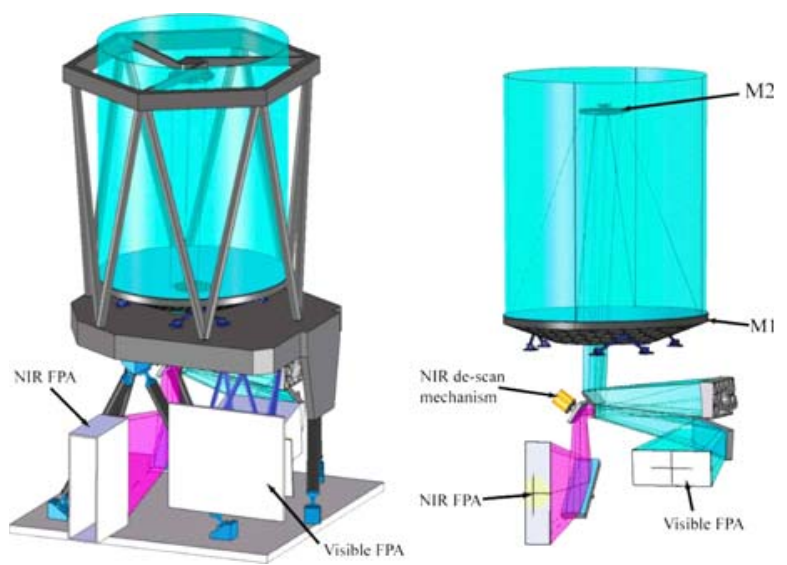

A primary driver for the GEO orbit choice is the high data rate- the orbit must be close enough to Earth to facilitate the transmission of the high amount of data produced by the payload every day (about 1.5 Tbits) given existing ground network facilities, while minimizing communication downlink time during which science observations cannot be made (with a fixed antenna).

The effects of the radiation environment at GEO, for both CCD bulk damage induced by solar protons and false detections induced by electrons with realistic shielding, is considered acceptable. However, DUNE specific radiation tests on CCD sensors will be required as an early development for confirming the measurement robustness to proton induced damage. A high elliptical orbit (HEO) operating beyond the radiation belt is an alternative in case electron radiation or thermal constraints prevent the use of GEO.

The payload for DUNE is a passively cooled $1.2 \mathrm{~m}$ diameter Korsch-like three-mirror telescope with two focal planes, visible and NIR covering 1 square degree. Figure 5 provides an overview of the payload. The Payload module design uses Silicon Carbide ( $\mathrm{SiC}$ ) technology for the telescope optics and structure. This provides low mass, high stability, low sensitivity to radiation and the ability to operate the entire instrument at cold temperature, typically below $170 \mathrm{~K}$, which will be important for cooling the large focal planes. The two FPAs, together with their passive cooling structures are isostatically mounted on the M1 baseplate. Also part of the payload are the de-scan mirror mechanism for the NIR channel and the additional payload data handling unit (PDHU).

\subsection{Telescope}

The telescope is a Korsch-like $f / 20$ three-mirror telescope. After the first two mirrors, the optical bundle is folded just after passing the primary mirror (M1) to reach the off-axis tertiary mirror. A dichroic element located near the exit pupil of the system provides the spectral separation of the visible and NIR channels. For the NIR, the de-scan mechanism close to the dichroic filter allows 
for a largely symmetric configuration of both spectral channels. The whole instrument fits within a cylinder of $1.8 \mathrm{~m}$ diameter and $2.65 \mathrm{~m}$ length. The payload mass is approximately $500 \mathrm{~kg}$, with $20 \%$ margin, and average/peak power estimates are 250/545 W.

Simulations have shown that the overall wavefront error (WFE) can be contained within $50 \mathrm{~nm}$ r.m.s, compatible with the required resolution. Distortion is limited to between $3-4 \mu \mathrm{m}$, introducing an $0.15 \mu \mathrm{rad}$ fixed (hence accessible to calibration) displacement in the object space. The need to have a calibration of the PSF shape error better than $0.1 \%$ over $50 \operatorname{arcmin}^{2}$ leads to a thermal stability of $\sim 10 \mathrm{mK}$ over $375 \mathrm{~s}$. Slow variations of solar incidence angle on the sunshield for DUNE will not significantly perturb the payload performance, even for angles as large as 30 degrees.

\subsection{Visible FPA}

The visible focal plane array (VFP) consists of 36 large format red-sensitive CCDs, arranged in a $9 \times 4$ array (Fig. 6) together with the associated mechanical support structure and electronics processing chains. Four additional CCDs dedicated to the AOCS measurements are located at the edge of the array. All CCDs are 4,096 pixel red-enhanced e2v CCD203-82 devices with square $12 \mu \mathrm{m}$ pixels. The physical size of the array is $466 \times$ $233 \mathrm{~mm}$ which corresponds to $1.09 \mathrm{deg} \times 0.52 \mathrm{deg}$. Each pixel is 0.102 arcsec, so that the PSF is well sampled in each direction over approximately 2.2 pixels, including all contributions. The VFP operates in the red band from $550-920 \mathrm{~nm}$. This bandpass is produced by the dichroic. The CCDs are fourphase devices, so they can be clocked in 1/4 pixel steps. The exposure duration on each CCD is $375 \mathrm{~s}$, permitting a slow readout rate and minimising readout

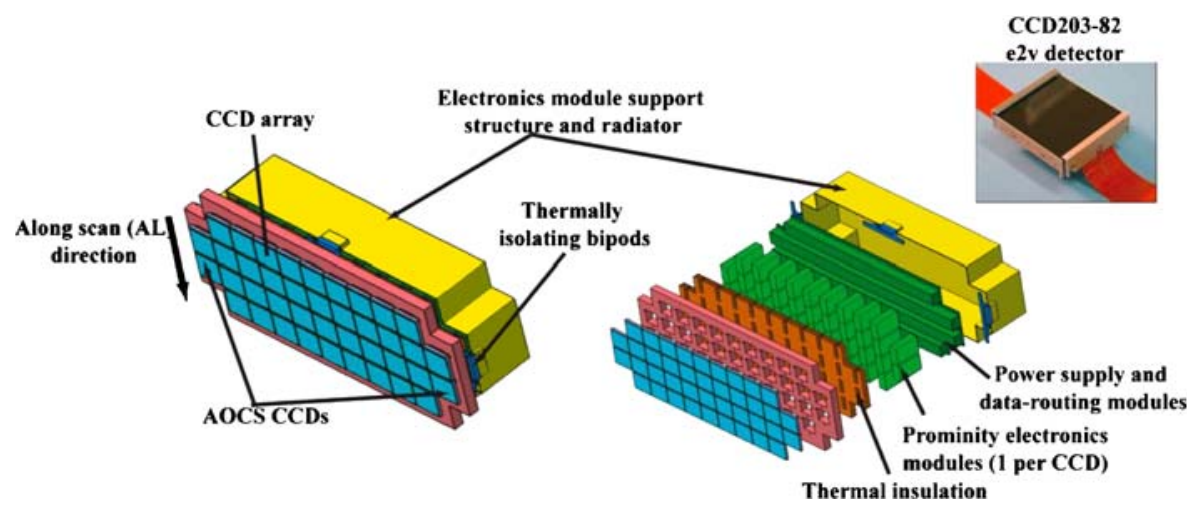

Fig. 6 Left the VFP assembly with the $9 \times 4$ array of CCDs and the four AOCS sensors on the front (blue) and the warm electronics radiator at the back (yellow). Right an expanded view of the VFP assembly, including the electronics modules and thermal hardware (but excluding the CCD radiator). Inset: The $22 \mathrm{v}$ CCD203-82 $4 \mathrm{k} \times 4 \mathrm{k}$ pixels shown here in a metal pack with flexi-leads for electrical connections. One of the flexi-leads will be removed 
noise. Combining four rows of CCDs will then provide a total exposure time of $1,500 \mathrm{~s}$.

The VFP will be used by the spacecraft in a closed-loop system to ensure that the scan rate and TDI clocking are synchronised. The two pairs of AOCS CCDs provide two speed measurements on relatively bright stars $(\mathrm{V} \sim 22-$ 23). The DUNE VFP is largely a self-calibrating instrument. For the shape measurements, stars of the appropriate magnitude will allow the PSF to be monitored for each CCD including the effects of optical distortion and detector alignment. Radiation-induced charge transfer inefficiency will modify the PSF and will also be self-calibrated in orbit.

\subsection{NIR FPA}

The NIR FPA consists of a $5 \times 12$ mosaic of 60 Hawaii 2 RG detector arrays from Teledyne, NIR bandpass filters for the wavelength bands Y, $\mathrm{J}$, and $\mathrm{H}$, the mechanical support structure, and the detector readout and signal processing electronics (see Fig. 7). The FPA is operated at a maximum temperature of $140 \mathrm{~K}$ for low dark current of $0.02 e^{-} / \mathrm{s}$. Each array has 2,048 $\times 2,048$ square pixels of $18 \mu \mathrm{m}$ size resulting in a $0.15 \times 0.15$ $\operatorname{arcsec}^{2}$ field of view (FOV) per pixel. The mosaic has a physical size of $482 \times 212 \mathrm{~mm}$, and covers a FOV of $1.04^{\circ} \times 0.44^{\circ}$ or 0.46 square degrees. The $\mathrm{HgCdTe}$ Hawaii $2 \mathrm{RG}$ arrays are standard devices sensitive in the 0.8 to $1.7 \mu \mathrm{m}$ wavelength range.

As the spacecraft is scanning the sky, the image motion on the NIR FPA is stabilised by a de-scanning mirror during the integration time of $300 \mathrm{~s}$ or less per NIR detector. The total integration time of $1,500 \mathrm{~s}$ for the $0.4^{\circ}$ high field is split among five rows and 3 wavelengths bands along the scan direction. The effective integration times are $600 \mathrm{~s}$ in $\mathrm{J}$ and $\mathrm{H}$, and $300 \mathrm{~s}$ in Y. For each array, the readout control, A/D conversion of the video output, and transfer of the digital data via a serial link is handled by the SIDECAR ASIC developed for JWST. To achieve the limiting magnitudes defined by the science requirements

Fig. 7 Layout of the NIR FPA (MPE/Kayser-Threde). The $5 \times 12$ Hawaii $2 R G$ Teledyne detector arrays (shown in the inset) are installed in a molybdenum structure

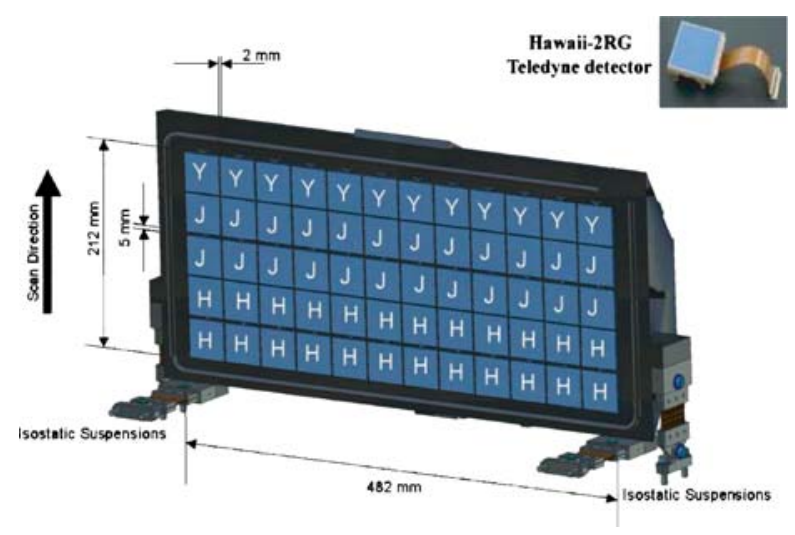


within these integration times, a minimum of 13 reads are required. Data are processed in the dedicated unit located in the service module.

\section{Basic spacecraft key factors}

The spacecraft platform architecture is fully based on well-proven and existing technologies. The mechanical, propulsion, and solar array systems are reused from Venus Express (ESA) and Mars-Express. All the AOCS, $\mu$-propulsion, Power control and DMS systems are reused from GAIA. Finally, the science telemetry system is a direct reuse from the PLEIADES (CNES) spacecraft. All TRLs are therefore high and all technologies are either standard or being developed for GAIA (AOCS for instance).

\subsection{Spacecraft architecture and configuration}

The spacecraft driving requirements are: (1) Passive cooling of both visible and NIR focal planes below $170 \mathrm{~K}$ and $140 \mathrm{~K}$, respectively; (2) the PSF stability requirement, which translates to line of sight (LOS) and payload thermal stability requirements; and (3) the high science data rate. The spacecraft consists of a Payload Module (PLM) that includes the instrument (telescope hardware, focal plane assemblies and on board science data management) and a Service Module (SVM). The SVM design is based on the Mars Express parallelepiped structure that is $1.7 \times 1.7 \times 1.4 \mathrm{~m}$, which accommodates all subsystems (propulsion, AOCS, communication, power, sunshield, etc) as well as the PLM. The spacecraft platform, and all technologies, are either standard ones or being developed into GAIA programme (e.g. AOCS).

\subsection{Sunshield and attitude control}

The nominal scan strategy assumes a constant, normal $(0 \mathrm{deg})$ incidence of the sun on the sunshield, while allowing a sun incidence angle of up to $30 \mathrm{deg}$ to provide margin, flexibility for data transmission manoeuvres and potential for further scan optimisation. The sunshield is a ribbed, MLI-covered central frame fixed to the platform. The satellite rotates in a draft scan-and-sweepback approach where the spacecraft is brought back to next scan position after each 20 deg strip. The scan rate is $1.12 \mathrm{deg}$ per hour, such that every day, one complete strip is scanned and transmitted to ground.

Due to the observation strategy and the fixed high gain antenna (HGA), the mission requires a high level of attitude manoeuvrability. During data collection, the spacecraft is rotated slowly about the sunshield axis. The slow scan control requirements are equivalent to three-axis satellite control. The line-of-sight stability requirement is $0.2 \mu \mathrm{rad}$ over $375 \mathrm{~s}$ (the integration time for one CCD) and is driven by optical quality and PSF smearing, and will be partially achieved using a continuous PSF calibration using the stars located 
in the neighborhood $\left(50 \operatorname{arcmin}^{2}\right)$ of each observed galaxy. Detailed analyses show that DUNE high pointing performance is comparable in difficulty to that achieved on GAIA during science observations. Similarly to GAIA, two pairs of dedicated CCD in the visible focal plane are used for measuring the spacecraft attitude speed vector. Hybridisation of the star tracker and payload measurements is used to reduce the noise injected by the star tracker in the loop. For all other operational phases and for the transition from coarse manoeuvres to the science observation mode, the attitude is controlled using the Mars Express propulsion system. The attitude estimation is based on using two star trackers (also used in science observing mode), six single-axis gyros and two sun sensors for monitoring DUNE pointing during manoeuvres with a typically accuracy better than 1 arcmin.

\subsection{Functional architecture: propulsion and electrical systems}

The star signal collected in the instrument is spread on the focal plane assembly and transformed into a digital electrical signal which is transferred to the Payload Data Handling Unit (PDHU), based on Thales/AlienaSpace heritage. Power management and regulation are performed by the Power Conditioning \& Distribution Unit (PCDU), and based on the GAIA program. Electrical power is generated by two solar arrays $\left(2.7 \mathrm{~m}^{2}\right.$ each), as used in the Mars Express and Venus Express ESA spacecraft. The control of their orientation is based on the orientation of the whole spacecraft towards the Sun. The panels are filled with AsGa cells.

The RF architecture is divided into two parts with the TT\&C system (SBand) plus a dedicated payload telemetry system (X-Band in the EES band (Earth Exploration Service). The allocated bandwidth for payload telemetry is $375 \mathrm{MHz}$ and high rate transmitters already exist for this purpose. The $\mathrm{X}$ band $155 \mathrm{Mbits} / \mathrm{s}$ TMTHD modulator can be reused from Pleiades spacecraft. A single fixed HGA of $30 \mathrm{~cm}$ diameter can be used (re-used from Venus Express). The RF power required is $25 \mathrm{~W}$, which also enables the re-use of the solid state power amplifier (SSPA) from Pleiades. The transmitted science data volume is estimated at 1.5 Tbits per day. The baseline approach consists in storing the science data on board in the PDHU, then to downlink the data twice per day. This can be achieved naturally twice per orbit at 06 and $18 \mathrm{~h}$ local time and using the rotation degree of freedom about the satellite-sun axis for orienting the antenna towards the ground station. The total transmission duration is less than $3 \mathrm{~h}$. The spacecraft attitude variation during transmission is less than $30 \mathrm{deg}$ (including AOCS margins). Twenty kilograms hydrazine propellant budget is required. In case the operational orbit would change to HEO, a dual frequency (S-Band + X-Band) $35 \mathrm{~m}$ ESOC antenna could fit with the mission needs, with in an increased HGA diameter $(70 \mathrm{~cm})$.

The required power on the GEO orbit is $1,055 \mathrm{~W}$. The sizing case is the science mode after eclipse with battery charging. Electrical power is generated by the two solar arrays of $2.7 \mathrm{~m}^{2}$ each. With a $30 \mathrm{deg}$ solar angle, the solar array 
can generate up to $1,150 \mathrm{~W}$ at the end of its life. The battery has been sized in a preliminary approach for the eclipse case (64 Ah need).

\section{Science operations and data processing}

The DUNE operational scenario follows the lines of a survey-type project. The satellite will operate autonomously except for defined ground contact periods during which housekeeping and science telemetry will be downlinked, and the commands needed to control spacecraft and payload will be uploaded. The DUNE processing pipeline is inspired by the Terapix pipeline used for the CFHT Legacy Survey. The total amount of science image data expected from DUNE is 370 Terapixels (TPx): $150 \mathrm{TPx}$ from the Wide, $120 \mathrm{TPx}$ for 3 months of the microlensing survey, $60 \mathrm{TPx}$ for the 3 months of the Galactic plane survey, and 40 TPx for 6 months deep survey. Based on previous experience, we estimate an equal amount of calibration data (flat fields, dark frames, etc.) will be taken over the course of the mission. This corresponds to 740 TB, roughly 20 times the amount of science data for CFHT during 20032007.

There are four main activities necessary for the data processing, handling, and data organisation of the DUNE surveys:

1. software development: image and catalogue processing, quality control, image and catalogue handling tools, pipeline development, survey monitoring, data archiving and distribution, numerical simulations, image simulations;

2. processing operation: running the pipeline, quality control and quality assessment operation and versioning, pipeline/software/database update and maintenance;

3. data archiving and data distribution: data and meta-data products and product description, public user interface, external data (non-DUNE) archiving and distribution, public outreach;

4. computing resources: data storage, cluster architecture, GRID technology.

\section{Conclusion: DUNE's impact on cosmology and astrophysics}

ESA's Planck mission will bring unprecedented precision to the measurement of the high redshift Universe. This will leave the dark energy dominated low redshift Universe as the next frontier in high precision cosmology. Constraints from the radiation perturbation in the high redshift $\mathrm{CMB}$, probed by Planck, combined with density perturbations at low redshifts, probed by DUNE, will form a complete set for testing all sectors of the cosmological model. In this respect, a DUNE+Planck programme can be seen as the next significant step in testing, and thus challenging, the standard model of cosmology. Table 2 illustrates just how precise the constraints on theory are expected to be: DUNE 
will offer high potential for ground-breaking discoveries of new physics, from dark energy to dark matter, initial conditions and the law of gravity. Our understanding of the Universe will be fundamentally altered in a post-DUNE era, with ESA's science programmes at the forefront of these discoveries. As described above, the science goals of DUNE go far beyond the measurement of dark energy. It is a mission which: (1) measures both effects of dark energy (i.e. the expansion history of the Universe and the growth of structure) by using weak lensing as the central probe; (2) places this high precision measurement of dark energy within a broader framework of high precision cosmology by constraining all sectors of the standard cosmology model (dark matter, initial conditions and Einstein gravity); (3) through a collection of unique legacy surveys is able to push the frontiers of the understanding of galaxy evolution and the physics of the local group; and finally (4) is able to obtain information on some of the lowest masses astronomy extrasolar planets, which could contain mirror Earths.

DUNE has been selected jointly with SPACE [5] in ESA's Cosmic Vision programme for an assessment phase which lead to the Euclid merged concept.

Acknowledgements We thank CNES for support on an earlier version of the DUNE mission and EADS/Astrium, Alcatel/Alenia Space, as well as Kayser/Threde for their help in the preparation of the ESA proposal.

\section{References}

1. Abdalla, et al.: arXiv:astro-ph/0705.1437 (2007)

2. Albrecht, A., et al.: arXiv:astro-ph/0609591 (2006)

3. Amara, A., Réfrégier, A.: MNRAS 381, 1018 (2007)

4. Amara, A., Refregier, A.: arXiv:astro-ph/0710.5171 (2007)

5. Cimatti, A., et al.: arXiv:0804.4433 (2008)

6. Douspis, M., Castro, P.G., Caprini, C., Aghanim, N.: arXiv:0802.0983. A\&A 485, 395 (2008)

7. Gavazzi, R., Treu, T., Koopmans, L.V.E., Bolton, A.S., Moustakas, L.A., Burles, S., Marshall, P.J.: APJ 677, 1046 (2008)

8. Kitching, T., et al.: arXiv:0801.4565 (2008)

9. Meneghetti, M., et al.: A\&A 482, 403 (2008)

10. Paulin-Henriksson, S., Amara, A., Voigt, L., Refregier, A., Bridle, S.L.: A\&A 484(1), 67-77 (2008)

11. Peacock, J.A., et al.: arXiv:astro-ph/0610906 (2006)

12. Refregier, A., et al.: Proc of the SPIE Symposium, 6265,58. Orlando (2006)

13. Grange, R., et al.: Proc of the SPIE Symposium, 6265,129. Orlando (2006)

14. Spergel, D., et al.: ApJS 170, 377 (2007) 